\title{
Comparison of Accrual Ratio and Cash Ratio Accuration in Financial Reports
}

\author{
Mahyudin, Sapto Hendri, Iman Waskito, *M. Ali Fikri \\ Faculty of Economy and Business, Mataram University. Jl. Majapahit No. 62, Mataram, \\ 83126, Indonesia \\ *Corresponding Author e-mail: fikri.sampala@unram.ac.id
}

Received: November 2021; Revised: December 2021; Published: December 2021

\begin{abstract}
Accounting and financial reporting fraud has been happening lately. Failure in estimating the veracity of financial reports starts from many aspects of accruals in the preparation of financial reports. This study seeks to explain the flow of financial transactions as important information, considering that activities in the financial sector require quick and relevant decisions. The flow of transactions in the financial reports consists of cash flows and accruals. Finance in business is almost similar to direct current and alternating current. In fact, this is misleading because of the ignorance of the readers of financial reports in interpreting profit, even though the misinterpretation will have an impact on investing errors. This study tries to analyze the results of financial ratio investments using the approach to the ratio of factors in the form of cash and accruals. Hermenuetics qualitative approach is used with data sourced from the Indonesia Stock Exchange. This study uses a sample of manufacturing companies listed on the Indonesia Stock Exchange. Three forms of financial ratio analysis are used, namely the analysis of ROA (return of assets), ROI (return of investment), and ROE (return of equity). Researchers measure using a comparison of ROA, ROI, and ROE based on accruals and cash. The results of the comparison of the accuracy of the accrual ratio and the cash ratio in the financial reports are presented further in this article.
\end{abstract}

Keywords: Financial ratios, accruals, cash, financial reports

How to Cite: Mahyudin, M., Hendri, S., Waskito, I., \& Fikri, M. (2021). Comparison of Accrual Ratio and Cash Ratio Accuration in Financial Reports. Prisma Sains : Jurnal Pengkajian Ilmu dan Pembelajaran Matematika dan IPA IKIP Mataram, 9(2), 402-412. doi:https://doi.org/10.33394/j-ps.v9i2.4609

https://doi.org/10.33394/j-ps.v9i2.4609

Copyright $\odot$ 2021, Mahyudin et al This is an open-access article under the CC-BY License.

\section{INTRODUCTION}

Every company has financial reports that aim to provide information regarding the financial position, performance, and changes in the financial position of a company that is useful for a large number of users of financial reports in making economic decisions (Maidoki, 2013). Financial reports must be prepared periodically for interested parties. Financial reports provide financial information of a company that can be used in making economic decisions and show the performance that has been carried out by management (stewardship) or management's responsibility for the use of the resources entrusted to it (Shakespeare, 2020). Financial reports are reports that describe the results of the accounting process that are used as a communication tool for parties with an interest in financial data or company activities.

The recent spike in fraud cases has caused the image of the accounting profession to decline. The phenomenon in the field also shows an increase in fraud cases in recent times, as stated in a previous study (Xin et al., 2018). Incidents like this in the world of accounting involve accrual elements (recognition), where accrual elements are very easy to engineer. To 
anticipate the impact of this, the accounting profession is taking steps by requiring companies to make cash-based financial reports which we know as cash flow reports (Osiichuk \& Mielcarz, 2021). As we all know that financial reports are the basis for making investment decisions, which then derives various types of analytical tools such as financial ratios, future financial estimates that are used for investment decision making.

Errors in financial reports are actually only known in detail by accountants, not other people. However, the fact is that other people also need a lot of things in financial reports, especially the use of financial ratios. No one can blame investors when they base their decision making based on financial ratios, but the important question is how accurately they can understand financial ratios that are sourced from financial reports that contain a lot of accrual elements, and when the financial ratios that are composed still contain accrual elements. This can lead to misinformation that can be fatal in investing. This study tries to analyze the problems contained in financial ratios when investors want to invest based on ratio analysis. The purpose of this study is to minimize the bias from the results of the ratio analysis for the purpose of estimating the accuracy of future investments.

The company's financial ratios are very important for a potential investor to determine how much investment can be given. The results of the analysis can also be used as a reference for business development (Minh-Trang et al., 2017). A ratio is one number that is compared to another number as a relationship. Financial ratio analysis is the process of observing indexes related to accounting in financial reports such as balance sheets, profit and loss reports and cash flow reports with the aim of assessing the financial performance of a company. This analysis is used to provide an overview of information about the company's financial position and performance that can be used as a guide in making business decisions (Krylov, 2018).

Financial ratio analysis is used by two main users, namely investors and management. Investors use financial ratios to see if the company is a good investment or not. By comparing financial ratios between companies and between industries, investors can determine which investment is the best. Meanwhile, management uses financial ratios to determine how well the company is performing to evaluate where the company can improve itself (Khalid et al., 2020). For example, if a company has low gross margins, managers can evaluate how to increase their gross margins. The general function of financial ratio analysis is useful for management and investors, among others: useful for a person/company who wants to invest in stocks; provide credit to a company; determine the level of health and development of the company, including the importance of evaluation; determine the level of competitor's financial strength (positioning); and determine the level of damage faced by the company.

There are several types of financial ratios, including: liquidity ratio, activity ratio, solvency ratio, profitability ratio, and investment ratio (Ponikvar et al., 2009). The liquidity ratio is a ratio that measures the short-term liquidity ability of a company by looking at the company's current assets relative to its current liabilities. Activa ratio looks at several assets and then determines what level of activity these assets are at a certain level of activity. Low activity at a certain level of sales will result in greater excess funds embedded in these assets. The excess funds would be better if invested in other more productive assets. Profitability ratio is a ratio that shows the level of return or gain (profit) compared to sales or assets. The investment ratio is a ratio that measures the company's ability to provide returns or rewards to funders, especially investors in the capital market within a certain period of time. This ratio has a value of benefit for investors according to the function of financial reports for investors to assess the performance of stock securities in the capital market (Ponikvar et al., 2009).

Investment can be interpreted as a commitment to invest a certain amount of funds at this time with the aim of obtaining profits in the future. In other words, investment is a commitment to sacrifice current consumption with the aim of increasing consumption in the 
future. Another understanding of investment is a form of investing funds or capital to generate wealth, which will be able to provide a profit rate of return (return) both now and in the future.

Expected future profits are compensation for the time and risk associated with the investment made. In the context of investment, the expected profit is often referred to as return (Brimberg et al., 2008). Basically the purpose of investors in investing is to maximize returns. There are several sources of risk that can affect the amount of investment risk, including: interest rate risk, business risk, financial risk (Duy Bui et al., 2021; Mouna \& Anis, 2016). To reduce risk, investors need to diversify. Diversification shows that investors need to form investment portfolios in such a way that risk can be minimized without reducing the expected return. Reducing risk without reducing return is the goal of investors in investing. Portfolio theory says don't put all the eggs in one basket, because if the basket falls, all the eggs in the basket will break. Likewise with the investments made, do not invest all of the funds in one form of investment, because when the investment fails, then all the invested funds may not return. This portfolio theory has taught the concept of portfolio diversification quantitatively. Portfolio is defined as a series of investment securities that are invested and held by investors, both individuals and entities. The combination of assets/assets can be in the form of real assets, financial assets or both (Beyhaghi \& Hawley, 2013).

Usually an investor in investing does not just choose one stock, but does a combination. The reason is that by combining stocks, investors can achieve optimal returns and at the same time can minimize risk through diversification (Okunevičiūtė-Neverauskienè et al., 2021). In other words, if an investor collects several securities to be used for investment, it means that the investor has formed a stock portfolio, the goal is to diversify in investment, which can minimize the risk faced by investors when compared to investing in individual stocks. However, choosing the optimal portfolio is not easy. Diversification is done to reduce portfolio risk, namely by combining or by adding investments (assets/assets/securities) that have a low negative or positive correlation so that the variability of returns or risk can be reduced.

Theories of thinking about investment cannot be separated from financial reports. Financial reports present data that still contains estimated values, such as depreciation, accounts payable, allowance for losses on receivables and other accrual items that lead to profit and loss reports. Moreover, it is coupled with the decreasing trust in the accounting profession due to the many cases of fraud. The next question is if the financial reports present a lot of estimated values, then the financial ratios that are sourced from financial reports automatically contain many estimates. In the end, users or most investors will experience 2 (two) times the estimation error. The first is the estimation error in the financial reports and the second is the error in the estimation of the ratio that is incorrectly sourced in the financial reports. In the end who can accept such a risk?

Let's move a little towards a more tangible basis, namely the cash basis, or better known as cash flow. To make cash flow reports, accountants need two important data, namely cash receipts records and cash disbursements records. Records of cash receipts are like cash income, investments made in cash. While cash disbursements records include expenses for paid expenses, expenses for investments with the aim of business expansion are also included in this activity. Cash flow reports are also made because they have many benefits that you will get. One of them is to see the financial position quickly and easily. Net cash flow shows a positive number, which means the company gets a profit or profit and vice versa if it shows a negative number it means the company has a deficit. In addition, cash flow reports can also be important information (supporting documents) to assess a company. Through cash flow reports, interested parties such as creditors, investors, and others can directly assess. 


\section{METHOD}

This study aims to determine the accuracy of cash and accrual-based financial ratios. This research is a research using a qualitative hermenuetics approach. The hermenuetics qualitative approach is intended to interpret the phenomena that occur in the field using secondary data from the Indonesia Stock Exchange. This study uses a sample of manufacturing companies listed on the Indonesia Stock Exchange, which are 183 companies. Data collection and analysis methods are almost the same as other research methods, this research uses a series of processes and techniques to extract data in the field. Data mining in the field, researchers do by means of secondary data analysis techniques in the capital market through analysis of financial reports from the side of the flow of financial ratio transactions.

There are 3 forms of financial ratios that are commonly used, namely the analysis of ROA (return of assets), ROI (return of investment), and ROE (return of equity). In this study, researchers measured using a comparison of ROA, ROI, and ROE based on accrual, and analysis of ROA, ROI, and ROE based on cash.

\section{Accrual-Based Financial Ratios}

- ROA Accrual = Accrual Profit $/$ Total Assets

- ROI Accrual $=$ Accrual Profit $/$ Total Fixed Assets

- ROE Accrual $=$ Accrual Profit $/$ Total Equity

\section{Cash-Based Financial Ratios}

- ROA Cash = Operating Cash Flow / Total Assets

- ROI Cash = Operating Cash Flow / Total Fixed Assets

- ROE Cash = Operating Cash Flow / Total Equity

The results of the analysis of the company's cash flow are processed, compared, and used as a basis for drawing conclusions which one is better between accrual-based and cashbased financial ratios.

\section{RESULTS AND DISCUSSION}

As previously explained, the purpose of this study is to determine the accuracy of cash and accrual-based financial ratios. Failure to estimate the correctness of financial reports starts from many aspects of accruals in the preparation of financial reports. This research explains that it needs to be done considering that activities in the financial sector require quick and relevant decisions. The flow of transactions in financial reports consisting of cash flows and accruals will be compared with capital market returns to determine investor response in interpreting investments based on financial ratio analysis.

This study uses a sample of manufacturing companies listed on the Indonesia Stock Exchange, which are 183 companies with a final result of 175 because a number of 8 companies have incomplete data. The following is presented research data.

Table 1. Research Data

\begin{tabular}{rlccccccc}
\hline No. & Code & $\begin{array}{c}\text { Return Share } \\
\text { Dec-2020 }\end{array}$ & ROA & $\begin{array}{c}\text { ROA } \\
\text { Cash }\end{array}$ & ROE & $\begin{array}{c}\text { ROE } \\
\text { Cash }\end{array}$ & ROI & $\begin{array}{c}\text { ROI } \\
\text { Cash }\end{array}$ \\
\hline 1 & TIRT & 0.02 & $(1.08)$ & $(0.10)$ & 1.09 & 0.10 & $(1.70)$ & $(0.16)$ \\
2 & GMFI & $(0.06)$ & $(0.63)$ & $(0.22)$ & 1.52 & 0.54 & $(1.44)$ & $(0.51)$ \\
3 & WSBP & $(0.04)$ & $(0.46)$ & 0.05 & $(4.16)$ & 0.49 & $(0.86)$ & 0.10 \\
4 & RMBA & $(0.02)$ & $(0.22)$ & 0.12 & $(0.47)$ & 0.27 & $(0.64)$ & 0.37 \\
5 & AMIN & - & $(0.17)$ & 0.03 & $(0.42)$ & 0.08 & $(0.54)$ & 0.10 \\
6 & ALMI & 0.02 & $(0.19)$ & 0.14 & 0.99 & $(0.72)$ & $(0.52)$ & 0.38 \\
7 & BATA & 0.01 & $(0.23)$ & 0.19 & $(0.37)$ & 0.31 & $(0.41)$ & 0.35 \\
\hline
\end{tabular}




\begin{tabular}{|c|c|c|c|c|c|c|c|c|}
\hline No. & Code & $\begin{array}{c}\text { Return Share } \\
\text { Dec-2020 }\end{array}$ & ROA & $\begin{array}{l}\text { ROA } \\
\text { Cash }\end{array}$ & ROE & $\begin{array}{l}\text { ROE } \\
\text { Cash }\end{array}$ & ROI & $\begin{array}{l}\text { ROI } \\
\text { Cash }\end{array}$ \\
\hline 8 & PCAR & $(0.06)$ & $(0.14)$ & 0.12 & $(0.23)$ & 0.19 & $(0.37)$ & 0.32 \\
\hline 9 & SULI & - & $(0.27)$ & 0.03 & 1.25 & $(0.15)$ & $(0.33)$ & 0.04 \\
\hline 10 & ADMG & $(0.06)$ & $(0.20)$ & $(0.01)$ & $(0.24)$ & $(0.01)$ & $(0.32)$ & $(0.01)$ \\
\hline 11 & RICY & 0.02 & $(0.05)$ & $(0.10)$ & $(0.22)$ & $(0.46)$ & $(0.28)$ & $(0.60)$ \\
\hline 12 & FOOD & $(0.02)$ & $(0.16)$ & 0.00 & $(0.32)$ & 0.01 & $(0.21)$ & 0.01 \\
\hline 13 & CNTX & $(0.01)$ & $(0.14)$ & 0.01 & 0.68 & $(0.03)$ & $(0.21)$ & 0.01 \\
\hline 14 & PURE & $(0.03)$ & $(0.14)$ & 0.01 & (1.62) & 0.13 & $(0.21)$ & 0.02 \\
\hline 15 & BIMA & - & $(0.16)$ & $(0.01)$ & (1.19) & $(0.09)$ & $(0.20)$ & $(0.02)$ \\
\hline 16 & POLY & 0.05 & $(0.09)$ & 0.06 & 0.02 & $(0.01)$ & $(0.18)$ & 0.12 \\
\hline 17 & LMPI & $(0.01)$ & $(0.06)$ & 0.04 & $(0.17)$ & 0.13 & $(0.18)$ & 0.13 \\
\hline 18 & ZONE & 0.01 & $(0.07)$ & 0.09 & $(0.15)$ & 0.19 & $(0.13)$ & 0.17 \\
\hline 19 & KICI & - & $(0.04)$ & 0.02 & $(0.08)$ & 0.03 & $(0.12)$ & 0.05 \\
\hline 20 & ARKA & $(0.07)$ & $(0.07)$ & $(0.00)$ & $(0.33)$ & $(0.02)$ & $(0.12)$ & $(0.01)$ \\
\hline 21 & BTEK & - & $(0.11)$ & $(0.00)$ & $(0.29)$ & $(0.00)$ & $(0.12)$ & $(0.00)$ \\
\hline 22 & LION & $(0.06)$ & $(0.03)$ & 0.01 & $(0.05)$ & 0.01 & $(0.12)$ & 0.03 \\
\hline 23 & PSDN & - & $(0.07)$ & $(0.04)$ & $(0.46)$ & $(0.23)$ & $(0.12)$ & $(0.06)$ \\
\hline 24 & BRNA & $(0.07)$ & $(0.09)$ & 0.05 & $(0.22)$ & 0.14 & $(0.12)$ & 0.07 \\
\hline 25 & GDYR & - & $(0.06)$ & 0.09 & $(0.16)$ & 0.23 & $(0.10)$ & 0.15 \\
\hline 26 & PICO & $(0.04)$ & $(0.06)$ & 0.03 & $(0.28)$ & 0.12 & $(0.10)$ & 0.04 \\
\hline 27 & MYTX & $(0.02)$ & $(0.07)$ & 0.02 & $(8.25)$ & 2.64 & (0.09) & 0.03 \\
\hline 28 & BELL & $(0.01)$ & $(0.03)$ & 0.11 & $(0.06)$ & 0.25 & $(0.08)$ & 0.32 \\
\hline 29 & LMSH & $(0.05)$ & $(0.04)$ & 0.01 & $(0.05)$ & 0.01 & $(0.08)$ & 0.01 \\
\hline 30 & AMFG & - & $(0.06)$ & 0.07 & $(0.16)$ & 0.19 & $(0.08)$ & 0.09 \\
\hline 31 & SCNP & 0.02 & $(0.04)$ & 0.06 & $(0.05)$ & 0.08 & $(0.08)$ & 0.12 \\
\hline 32 & ARGO & 0.02 & $(0.07)$ & $(0.03)$ & 0.06 & 0.03 & $(0.07)$ & $(0.03)$ \\
\hline 33 & TCID & $(0.00)$ & $(0.03)$ & 0.13 & $(0.04)$ & 0.17 & $(0.07)$ & 0.32 \\
\hline 34 & KIAS & - & $(0.05)$ & 0.02 & $(0.06)$ & 0.03 & $(0.07)$ & 0.03 \\
\hline 35 & GDST & (0.06) & $(0.05)$ & 0.00 & (0.09) & 0.00 & $(0.07)$ & 0.00 \\
\hline 36 & IKAI & - & $(0.06)$ & $(0.01)$ & (0.09) & $(0.02)$ & $(0.06)$ & $(0.01)$ \\
\hline 37 & KBLI & $(0.04)$ & $(0.01)$ & 0.25 & $(0.01)$ & 0.32 & $(0.06)$ & 1.50 \\
\hline 38 & SSTM & 0.02 & $(0.03)$ & 0.07 & $(0.08)$ & 0.19 & $(0.06)$ & 0.15 \\
\hline 39 & MRAT & $(0.03)$ & $(0.01)$ & $(0.03)$ & $(0.02)$ & $(0.05)$ & $(0.06)$ & $(0.14)$ \\
\hline 40 & FPNI & 0.10 & $(0.03)$ & 0.03 & $(0.04)$ & 0.04 & $(0.05)$ & 0.05 \\
\hline 41 & ERTX & $(0.02)$ & $(0.02)$ & 0.06 & $(0.07)$ & 0.24 & $(0.04)$ & 0.13 \\
\hline 42 & IKAN & $(0.01)$ & $(0.01)$ & $(0.30)$ & $(0.02)$ & $(0.57)$ & $(0.04)$ & (1.27) \\
\hline 43 & INCF & 0.04 & $(0.01)$ & $(0.04)$ & $(0.05)$ & $(0.15)$ & $(0.03)$ & $(0.11)$ \\
\hline 44 & CTBN & $(0.06)$ & $(0.01)$ & 0.09 & $(0.02)$ & 0.12 & $(0.03)$ & 0.22 \\
\hline 45 & IKBI & $(0.01)$ & $(0.02)$ & 0.10 & $(0.02)$ & 0.14 & $(0.03)$ & 0.18 \\
\hline 46 & POLU & 0.07 & $(0.01)$ & 0.14 & $(0.02)$ & 0.24 & $(0.03)$ & 0.29 \\
\hline 47 & APLI & $(0.01)$ & $(0.02)$ & 0.09 & $(0.03)$ & 0.19 & $(0.03)$ & 0.14 \\
\hline 48 & BRAM & $(0.02)$ & $(0.02)$ & 0.01 & $(0.02)$ & 0.01 & $(0.03)$ & 0.02 \\
\hline 49 & INOV & $(0.06)$ & $(0.01)$ & 0.01 & $(0.03)$ & 0.03 & $(0.02)$ & 0.02 \\
\hline 50 & ESTII & $(0.02)$ & $(0.01)$ & 0.08 & $(0.04)$ & 0.33 & $(0.02)$ & 0.15 \\
\hline 51 & MAIN & $(0.05)$ & $(0.01)$ & 0.06 & $(0.02)$ & 0.14 & $(0.02)$ & 0.11 \\
\hline 52 & SBAT & 0.22 & $(0.01)$ & 0.04 & $(0.03)$ & 0.14 & $(0.02)$ & 0.07 \\
\hline 53 & AUTO & $(0.02)$ & $(0.01)$ & 0.08 & $(0.01)$ & 0.10 & $(0.01)$ & 0.11 \\
\hline 54 & ALTO & $(0.03)$ & $(0.01)$ & 0.03 & $(0.02)$ & 0.08 & $(0.01)$ & 0.03 \\
\hline 55 & INAF & $(0.00)$ & $(0.00)$ & 0.03 & $(0.01)$ & 0.11 & $(0.01)$ & 0.08 \\
\hline
\end{tabular}




\begin{tabular}{|c|c|c|c|c|c|c|c|c|}
\hline No. & Code & $\begin{array}{c}\text { Return Share } \\
\text { Dec-2020 }\end{array}$ & ROA & $\begin{array}{l}\text { ROA } \\
\text { Cash }\end{array}$ & ROE & $\begin{array}{l}\text { ROE } \\
\text { Cash }\end{array}$ & ROI & $\begin{array}{l}\text { ROI } \\
\text { Cash }\end{array}$ \\
\hline 56 & KAEF & $(0.00)$ & $(0.00)$ & 0.06 & $(0.01)$ & 0.14 & $(0.01)$ & 0.09 \\
\hline 57 & PRAS & - & $(0.00)$ & 0.00 & $(0.01)$ & 0.00 & $(0.00)$ & 0.00 \\
\hline 58 & TFCO & $(0.00)$ & $(0.00)$ & 0.07 & $(0.00)$ & 0.07 & $(0.00)$ & 0.10 \\
\hline 59 & TRIS & 0.28 & $(0.00)$ & 0.08 & $(0.00)$ & 0.13 & $(0.00)$ & 0.22 \\
\hline 60 & GGRP & 0.05 & $(0.00)$ & 0.23 & $(0.00)$ & 0.34 & $(0.00)$ & 0.29 \\
\hline 61 & CINT & $(0.01)$ & $(0.00)$ & 0.02 & $(0.00)$ & 0.03 & $(0.00)$ & 0.04 \\
\hline 62 & PANI & 0.06 & 0.00 & $(0.06)$ & 0.00 & $(0.14)$ & 0.00 & $(0.22)$ \\
\hline 63 & SMBR & $(0.05)$ & 0.00 & 0.07 & 0.00 & 0.12 & 0.00 & 0.09 \\
\hline 64 & VOKS & $(0.06)$ & 0.00 & $(0.05)$ & 0.00 & $(0.12)$ & 0.00 & $(0.19)$ \\
\hline 65 & SWAT & 0.07 & 0.00 & 0.00 & 0.01 & 0.01 & 0.01 & 0.01 \\
\hline 66 & KBLM & $(0.02)$ & 0.00 & 0.06 & 0.01 & 0.08 & 0.01 & 0.09 \\
\hline 67 & SKBM & 0.01 & 0.00 & 0.01 & 0.01 & 0.02 & 0.01 & 0.02 \\
\hline 68 & INRU & - & 0.01 & $(0.02)$ & 0.02 & $(0.05)$ & 0.01 & $(0.02)$ \\
\hline 69 & ENZO & - & 0.00 & $(0.13)$ & 0.01 & $(0.24)$ & 0.01 & $(0.30)$ \\
\hline 70 & SOFA & 0.04 & 0.00 & 0.29 & 0.01 & 0.42 & 0.01 & 0.73 \\
\hline 71 & SINI & $(0.01)$ & 0.00 & 0.02 & 0.02 & 0.10 & 0.01 & 0.04 \\
\hline 72 & KPAS & (0.07) & 0.01 & 0.06 & 0.02 & 0.11 & 0.01 & 0.08 \\
\hline 73 & TALF & $(0.04)$ & 0.01 & 0.04 & 0.01 & 0.06 & 0.01 & 0.07 \\
\hline 74 & CAKK & - & 0.01 & 0.06 & 0.02 & 0.09 & 0.01 & 0.08 \\
\hline 75 & TOTO & $(0.01)$ & 0.01 & 0.10 & 0.02 & 0.16 & 0.02 & 0.18 \\
\hline 76 & EPAC & $(0.02)$ & 0.01 & $(0.02)$ & 0.02 & $(0.04)$ & 0.02 & $(0.03)$ \\
\hline 77 & AGII & 0.04 & 0.01 & 0.05 & 0.03 & 0.11 & 0.02 & 0.07 \\
\hline 78 & INDR & $(0.04)$ & 0.01 & 0.02 & 0.02 & 0.04 & 0.02 & 0.03 \\
\hline 79 & KRAS & $(0.03)$ & 0.02 & 0.02 & 0.12 & 0.16 & 0.02 & 0.03 \\
\hline 80 & BRPT & $(0.04)$ & 0.02 & 0.06 & 0.04 & 0.15 & 0.02 & 0.08 \\
\hline 81 & TPIA & $(0.03)$ & 0.01 & 0.11 & 0.03 & 0.21 & 0.02 & 0.19 \\
\hline 82 & DPNS & 0.05 & 0.01 & 0.04 & 0.01 & 0.04 & 0.03 & 0.09 \\
\hline 83 & $\mathrm{COCO}$ & $(0.07)$ & 0.01 & $(0.12)$ & 0.02 & $(0.29)$ & 0.03 & $(0.32)$ \\
\hline 84 & JECC & (0.07) & 0.01 & 0.27 & 0.02 & 0.55 & 0.03 & 0.70 \\
\hline 85 & SIPD & - & 0.01 & 0.05 & 0.03 & 0.14 & 0.03 & 0.13 \\
\hline 86 & INDS & $(0.00)$ & 0.02 & 0.11 & 0.02 & 0.12 & 0.03 & 0.17 \\
\hline 87 & MLIA & $(0.06)$ & 0.03 & 0.07 & 0.05 & 0.15 & 0.03 & 0.09 \\
\hline 88 & $\mathrm{CBMF}$ & $(0.01)$ & 0.02 & $(0.08)$ & 0.02 & $(0.12)$ & 0.04 & $(0.20)$ \\
\hline 89 & LPIN & $(0.05)$ & 0.02 & 0.05 & 0.02 & 0.05 & 0.04 & 0.09 \\
\hline 90 & BUDI & (0.04) & 0.02 & 0.07 & 0.05 & 0.15 & 0.04 & 0.11 \\
\hline 91 & WTON & (0.04) & 0.01 & 0.09 & 0.04 & 0.24 & 0.04 & 0.25 \\
\hline 92 & SMGR & $(0.01)$ & 0.03 & 0.09 & 0.06 & 0.20 & 0.04 & 0.12 \\
\hline 93 & SMCB & 0.02 & 0.03 & 0.05 & 0.08 & 0.13 & 0.04 & 0.06 \\
\hline 94 & ESIP & $(0.06)$ & 0.02 & 0.02 & 0.03 & 0.03 & 0.04 & 0.03 \\
\hline 95 & TRST & 0.00 & 0.03 & 0.08 & 0.05 & 0.14 & 0.04 & 0.11 \\
\hline 96 & AKPI & $(0.01)$ & 0.03 & 0.09 & 0.05 & 0.18 & 0.04 & 0.14 \\
\hline 97 & KINO & - & 0.02 & $(0.01)$ & 0.04 & $(0.03)$ & 0.04 & $(0.03)$ \\
\hline 98 & SMKL & $(0.02)$ & 0.02 & 0.08 & 0.05 & 0.19 & 0.04 & 0.15 \\
\hline 99 & JSKY & 0.02 & 0.02 & $(0.07)$ & 0.03 & $(0.14)$ & 0.04 & $(0.18)$ \\
\hline 100 & GOOD & 0.03 & 0.03 & 0.13 & 0.07 & 0.28 & 0.04 & 0.19 \\
\hline 101 & ROTI & 0.02 & 0.03 & 0.11 & 0.05 & 0.15 & 0.05 & 0.17 \\
\hline 102 & PTSN & $(0.03)$ & 0.03 & 0.09 & 0.05 & 0.14 & 0.05 & 0.13 \\
\hline 103 & PEHA & - & 0.03 & 0.14 & 0.07 & 0.35 & 0.06 & 0.28 \\
\hline
\end{tabular}




\begin{tabular}{|c|c|c|c|c|c|c|c|c|}
\hline No. & Code & $\begin{array}{c}\text { Return Share } \\
\text { Dec-2020 }\end{array}$ & ROA & $\begin{array}{l}\text { ROA } \\
\text { Cash }\end{array}$ & ROE & $\begin{array}{l}\text { ROE } \\
\text { Cash }\end{array}$ & ROI & $\begin{array}{l}\text { ROI } \\
\text { Cash }\end{array}$ \\
\hline 104 & JPFA & $(0.04)$ & 0.03 & 0.16 & 0.07 & 0.36 & 0.06 & 0.29 \\
\hline 105 & TOYS & - & 0.00 & $(0.51)$ & 0.00 & $(0.70)$ & 0.06 & (11.34) \\
\hline 106 & GJTL & - & 0.03 & 0.15 & 0.09 & 0.38 & 0.06 & 0.26 \\
\hline 107 & IMAS & $(0.00)$ & 0.04 & 0.05 & 0.15 & 0.20 & 0.06 & 0.08 \\
\hline 108 & TKIM & $(0.04)$ & 0.05 & 0.06 & 0.10 & 0.11 & 0.06 & 0.08 \\
\hline 109 & INKP & $(0.02)$ & 0.03 & 0.06 & 0.07 & 0.13 & 0.07 & 0.13 \\
\hline 110 & INDF & 0.00 & 0.06 & 0.08 & 0.12 & 0.18 & 0.07 & 0.11 \\
\hline 111 & IPOL & $(0.06)$ & 0.04 & 0.12 & 0.07 & 0.20 & 0.07 & 0.21 \\
\hline 112 & IMPC & $(0.02)$ & 0.04 & 0.08 & 0.07 & 0.15 & 0.07 & 0.16 \\
\hline 113 & HOKI & $(0.02)$ & 0.04 & 0.09 & 0.06 & 0.12 & 0.08 & 0.16 \\
\hline 114 & ISSP & $(0.06)$ & 0.04 & 0.06 & 0.07 & 0.11 & 0.08 & 0.12 \\
\hline 115 & NIKL & $(0.04)$ & 0.02 & 0.06 & 0.06 & 0.18 & 0.08 & 0.26 \\
\hline 116 & ASII & $(0.00)$ & 0.05 & 0.11 & 0.09 & 0.19 & 0.08 & 0.18 \\
\hline 117 & YPAS & $(0.07)$ & 0.04 & $(0.02)$ & 0.08 & $(0.03)$ & 0.09 & $(0.04)$ \\
\hline 118 & ICBP & 0.03 & 0.07 & 0.09 & 0.15 & 0.19 & 0.09 & 0.11 \\
\hline 119 & SKLT & - & 0.05 & 0.13 & 0.09 & 0.25 & 0.09 & 0.25 \\
\hline 120 & SPMA & $(0.04)$ & 0.07 & 0.20 & 0.10 & 0.30 & 0.09 & 0.28 \\
\hline 121 & INAI & 0.01 & 0.02 & 0.02 & 0.09 & 0.07 & 0.09 & 0.08 \\
\hline 122 & KDSI & $(0.01)$ & 0.04 & 0.07 & 0.08 & 0.14 & 0.10 & 0.16 \\
\hline 123 & IFII & $(0.03)$ & 0.07 & 0.17 & 0.07 & 0.18 & 0.10 & 0.25 \\
\hline 124 & CCSI & $(0.01)$ & 0.04 & 0.09 & 0.06 & 0.13 & 0.10 & 0.21 \\
\hline 125 & ALKA & $(0.05)$ & 0.00 & $(0.26)$ & 0.01 & (1.01) & 0.10 & (10.00) \\
\hline 126 & UCID & $(0.03)$ & 0.04 & 0.18 & 0.07 & 0.30 & 0.10 & 0.43 \\
\hline 127 & MASA & $(0.03)$ & 0.07 & 0.13 & 0.14 & 0.25 & 0.11 & 0.19 \\
\hline 128 & BTON & $(0.05)$ & 0.02 & 0.09 & 0.02 & 0.11 & 0.11 & 0.51 \\
\hline 129 & INTP & 0.01 & 0.06 & 0.13 & 0.08 & 0.16 & 0.12 & 0.24 \\
\hline 130 & MDKI & (0.06) & 0.08 & 0.06 & 0.09 & 0.07 & 0.12 & 0.10 \\
\hline 131 & SCCO & - & 0.06 & 0.32 & 0.07 & 0.37 & 0.12 & 0.64 \\
\hline 132 & CLEO & $(0.03)$ & 0.10 & 0.17 & 0.15 & 0.25 & 0.12 & 0.21 \\
\hline 133 & DMND & 0.01 & 0.05 & 0.09 & 0.06 & 0.11 & 0.13 & 0.25 \\
\hline 134 & WOOD & - & 0.06 & 0.03 & 0.11 & 0.06 & 0.13 & 0.07 \\
\hline 135 & CAMP & $(0.01)$ & 0.04 & 0.19 & 0.05 & 0.21 & 0.13 & 0.60 \\
\hline 136 & KMTR & - & 0.05 & $(0.12)$ & 0.12 & $(0.28)$ & 0.14 & $(0.34)$ \\
\hline 137 & ALDO & 0.02 & 0.06 & 0.13 & 0.10 & 0.20 & 0.15 & 0.29 \\
\hline 138 & TBMS & $(0.01)$ & 0.03 & 0.19 & 0.09 & 0.58 & 0.15 & 0.99 \\
\hline 139 & INCI & $(0.05)$ & 0.07 & 0.11 & 0.09 & 0.14 & 0.16 & 0.24 \\
\hline 140 & PBRX & $(0.02)$ & 0.03 & $(0.05)$ & 0.07 & $(0.11)$ & 0.17 & $(0.27)$ \\
\hline 141 & MLBI & 0.01 & 0.10 & 0.30 & 0.20 & 0.61 & 0.17 & 0.51 \\
\hline 142 & EKAD & $(0.00)$ & 0.09 & 0.21 & 0.10 & 0.24 & 0.19 & 0.44 \\
\hline 143 & MOLI & - & 0.10 & $(0.03)$ & 0.16 & $(0.05)$ & 0.19 & $(0.06)$ \\
\hline 144 & $\mathrm{SOHO}$ & 0.00 & 0.04 & 0.14 & 0.07 & 0.26 & 0.21 & 0.75 \\
\hline 145 & CPIN & $(0.01)$ & 0.12 & 0.16 & 0.16 & 0.21 & 0.22 & 0.27 \\
\hline 146 & DVLA & 0.00 & 0.07 & 0.05 & 0.10 & 0.08 & 0.24 & 0.18 \\
\hline 147 & SRSN & $(0.02)$ & 0.10 & 0.01 & 0.15 & 0.01 & 0.26 & 0.02 \\
\hline 148 & GGRM & $(0.00)$ & 0.10 & 0.22 & 0.13 & 0.30 & 0.26 & 0.61 \\
\hline 149 & TSPC & 0.02 & 0.09 & 0.11 & 0.13 & 0.15 & 0.27 & 0.31 \\
\hline 150 & BAJA & $(0.06)$ & 0.07 & 0.28 & 0.44 & 1.67 & 0.28 & 1.08 \\
\hline 151 & MYOR & $(0.07)$ & 0.10 & 0.19 & 0.18 & 0.33 & 0.29 & 0.54 \\
\hline
\end{tabular}




\begin{tabular}{clccccccc}
\hline No. & Code & $\begin{array}{c}\text { Return Share } \\
\text { Dec-2020 }\end{array}$ & ROA & $\begin{array}{c}\text { ROA } \\
\text { Cash }\end{array}$ & ROE & $\begin{array}{c}\text { ROE } \\
\text { Cash }\end{array}$ & ROI & $\begin{array}{c}\text { ROI } \\
\text { Cash }\end{array}$ \\
\hline 152 & KLBF & $(0.01)$ & 0.13 & 0.19 & 0.16 & 0.23 & 0.30 & 0.44 \\
153 & MERK & 0.00 & 0.08 & 0.08 & 0.13 & 0.12 & 0.31 & 0.29 \\
154 & STTP & - & 0.18 & 0.27 & 0.23 & 0.35 & 0.32 & 0.48 \\
155 & ADES & $(0.03)$ & 0.14 & 0.24 & 0.19 & 0.33 & 0.33 & 0.56 \\
156 & PYFA & 0.01 & 0.14 & 0.00 & 0.21 & 0.01 & 0.33 & 0.01 \\
157 & SAMF & 0.01 & 0.09 & 0.17 & 0.15 & 0.30 & 0.35 & 0.68 \\
158 & ULTJ & $(0.04)$ & 0.13 & 0.14 & 0.24 & 0.25 & 0.36 & 0.39 \\
159 & SLIS & $(0.02)$ & 0.07 & $(0.04)$ & 0.14 & $(0.08)$ & 0.39 & $(0.22)$ \\
160 & PBID & 0.03 & 0.15 & 0.22 & 0.19 & 0.28 & 0.40 & 0.57 \\
161 & ARNA & 0.04 & 0.16 & 0.21 & 0.24 & 0.32 & 0.40 & 0.53 \\
162 & MARK & 0.08 & 0.20 & 0.30 & 0.36 & 0.53 & 0.40 & 0.60 \\
163 & IGAR & - & 0.10 & 0.11 & 0.11 & 0.13 & 0.41 & 0.49 \\
164 & MBTO & 0.01 & 0.36 & 0.00 & 0.60 & 0.00 & 0.44 & 0.00 \\
165 & UNIC & $(0.03)$ & 0.12 & 0.27 & 0.14 & 0.32 & 0.48 & 1.09 \\
166 & WIIM & $(0.02)$ & 0.10 & 0.13 & 0.13 & 0.18 & 0.49 & 0.66 \\
167 & SMSM & 0.03 & 0.16 & 0.28 & 0.21 & 0.36 & 0.51 & 0.87 \\
168 & SIDO & - & 0.24 & 0.27 & 0.29 & 0.32 & 0.52 & 0.58 \\
169 & UNVR & - & 0.34 & 0.41 & 1.43 & 1.69 & 0.60 & 0.71 \\
170 & CEKA & $(0.02)$ & 0.12 & 0.11 & 0.15 & 0.14 & 0.63 & 0.57 \\
171 & AISA & $(0.07)$ & 0.60 & $(0.03)$ & 1.46 & $(0.07)$ & 0.92 & $(0.04)$ \\
172 & DLTA & 0.04 & 0.10 & 0.20 & 0.12 & 0.24 & 0.97 & 2.03 \\
173 & HMSP & $(0.01)$ & 0.17 & 0.24 & 0.28 & 0.40 & 0.99 & 1.39 \\
174 & HRTA & $(0.05)$ & 0.06 & $(0.23)$ & 0.13 & $(0.48)$ & 1.06 & $(4.02)$ \\
175 & STAR & $(0.01)$ & 0.01 & 0.17 & 0.01 & 0.17 & 5.56 & 81.50 \\
\hline
\end{tabular}

There are 3 forms of ratios used in analyzing financial ratios to stock returns, namely analysis of ROA, ROI, and ROE based on accruals, and analysis of ROA, ROI, and ROE based on cash. The following is the result of the comparison of stock returns (investment) based on a comparison chart.

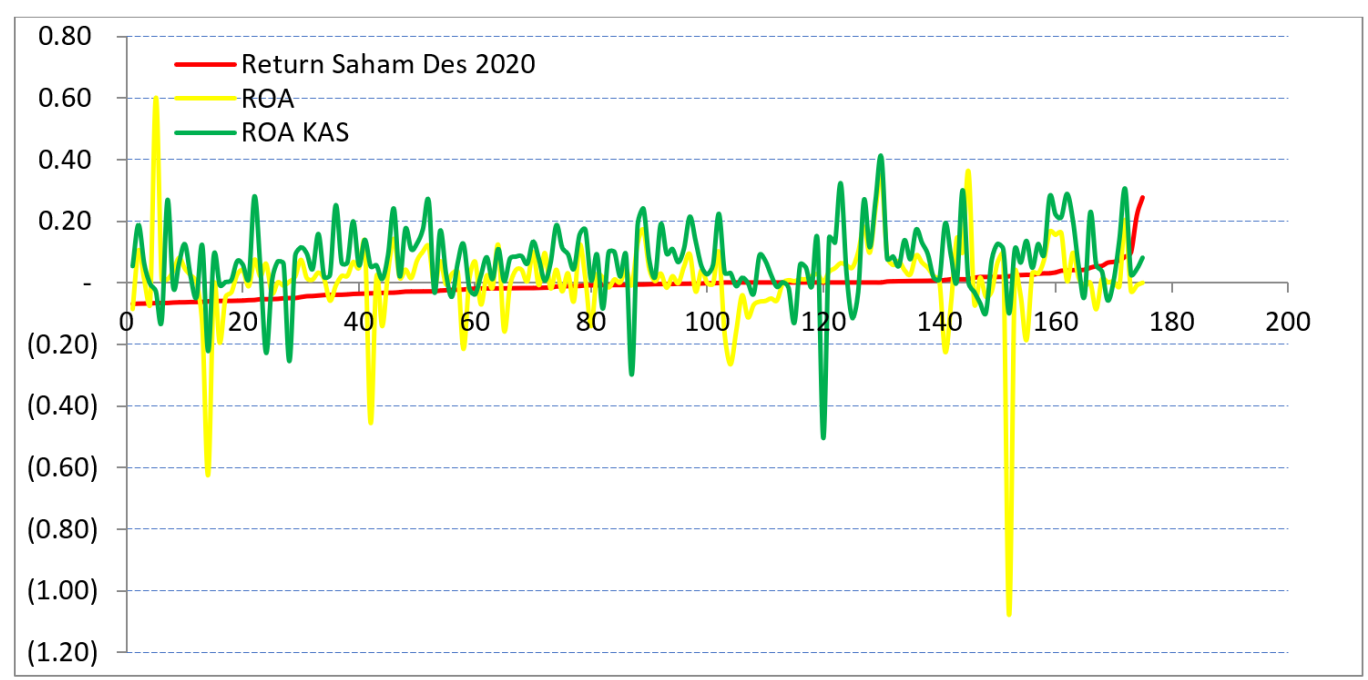

Figure 1. Graph of ROA Accrual and ROA Cash

The results of the comparison using the ROA accrual and ROA cash approach (Figure 1) show a unidirectional relationship, namely an increase in ROA accruals followed by an 
increase in ROA cash. Thus, when viewed from the trend of the stock return chart, the chart tends to follow the movement pattern of the cash ROA basis.

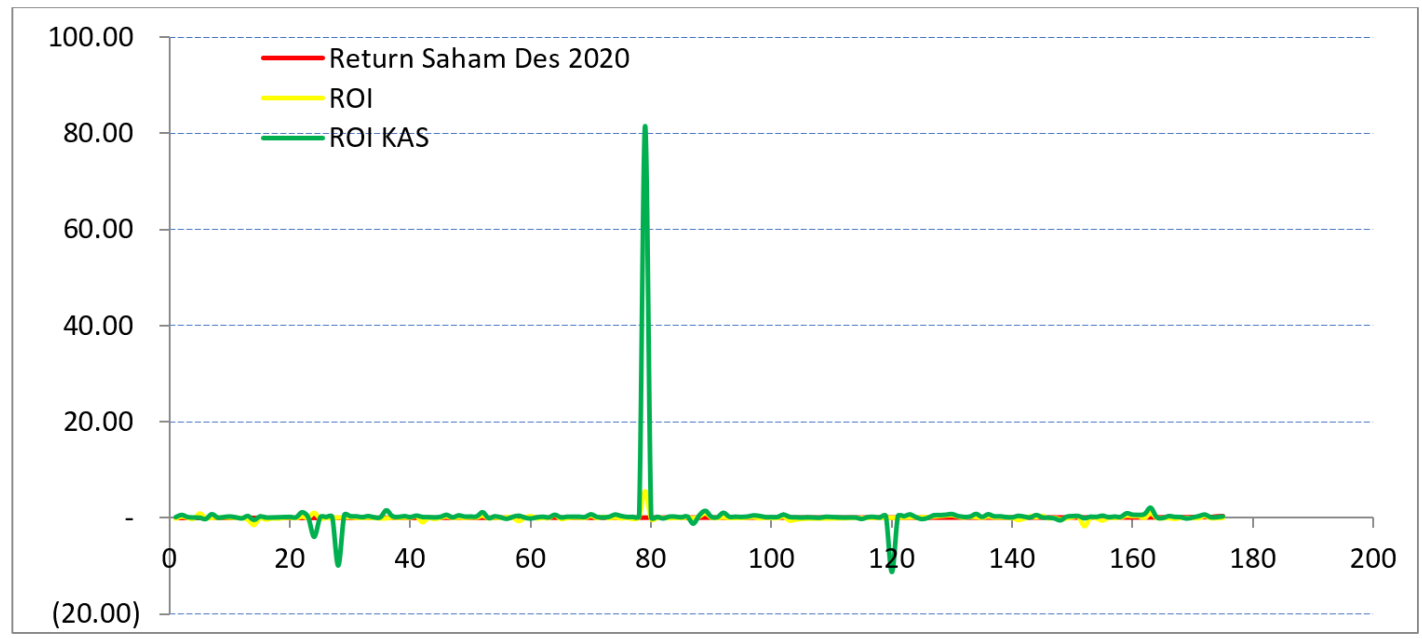

Figure 2. Graph of ROI Accrual and ROI Cash

The results of the comparison using the ROI accrual and ROI cash approach show a unidirectional relationship (Figure 2), namely an increase in ROI accruals followed by an increase in ROI cash. When viewed from the trend of the stock return chart, the stock return chart tends to follow the movement pattern of ROI accruals and ROI cash.

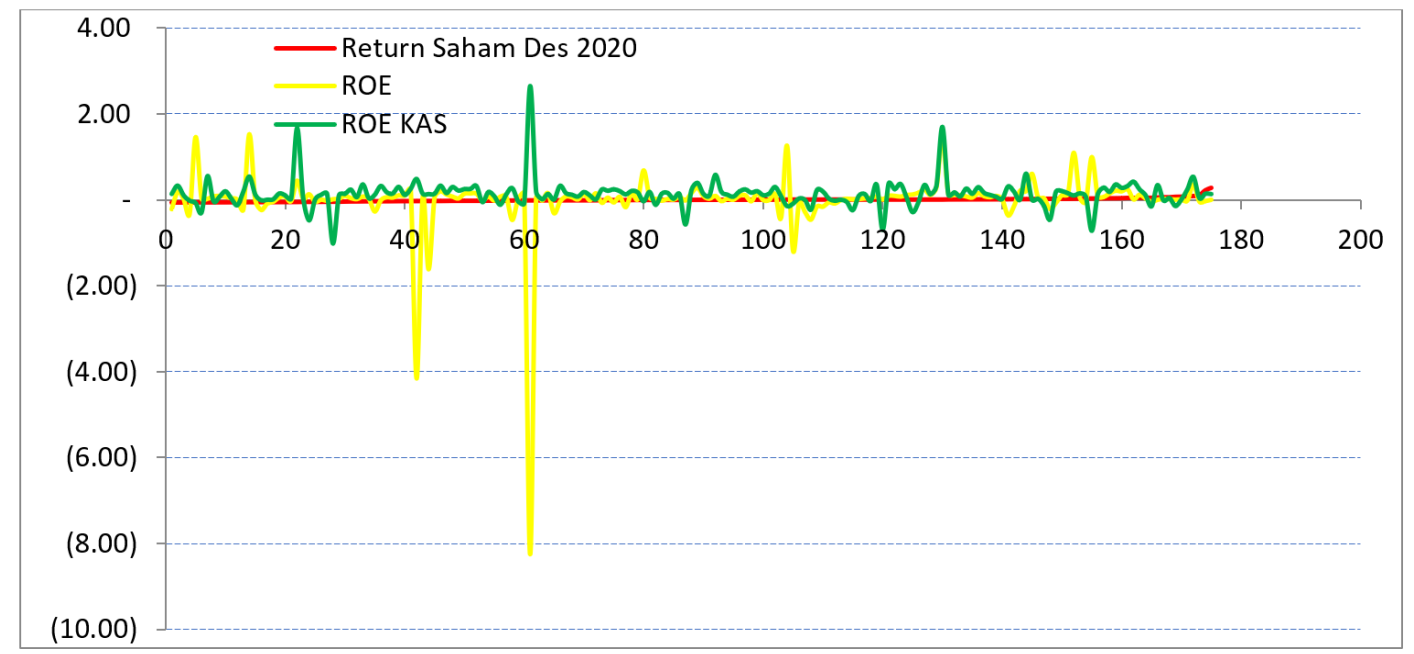

Figure 3. Graph of ROE Accrual and ROE Cash

Comparison using the ROE accrual and ROE cash approach shows an inverse relationship (Figure 3), namely when accrual ROE increases, cash ROA decreases. However, when viewed from the trend of the stock return chart, the chart tends to follow the movement pattern of the cash ROE basis.

The results of this study support the theory of thought that investment cannot be separated from financial reports, where financial reports present data that still contains estimated values, such as depreciation, accounts payable, reserves for losses on receivables and other accrual items that lead to profit and loss reports (Tran \& Duong , 2020). Moreover, coupled with the decreasing trust in the accounting profession due to the many cases of fraud, so that if the financial reports present a lot of estimated values, the financial ratios that are sourced from financial reports automatically contain many estimates. In the end, users or 
most investors will experience 2 (two) times the estimation error. The first is the estimation error in the financial reports and the second is the error in the estimation of the ratio that is incorrectly sourced in the financial reports. So that investors rely more on reports that are not estimating, but certain, namely the cash approach.

\section{CONCLUSION}

Financial ratio analysis studies on stock returns have been carried out, namely analysis of ROA, ROI, and ROE based on accruals, and analysis of ROA, ROI, and ROE based on cash. The ROA accrual approach and cash ROA show a unidirectional relationship, namely an increase in accrual ROA followed by an increase in cash ROA. Likewise, the accrual ROI and cash ROI approaches show a unidirectional relationship. However, the ROE accrual approach and cash ROE show an inverse relationship, namely when accrual ROE increases, cash ROA decreases. The results of the study provide evidence that the financial ratio approach based on the Cash approach provides more useful information for investors in investing. There are several shortcomings in this study, namely this study only uses one year of observation data and uses manufacturing companies. It is suggested that the next research can use a different company with a longer observation period.

\section{RECOMMENDATION}

Theoretically, the results of this study provide a theoretical contribution that the cash approach is more useful than accrual. Practically, this research provides a discourse for investors to conduct cash analysis. Finally, it is expected that policy makers such as the government will prioritize the cash approach in decision making.

\section{ACKNOWLEDGMENT}

The author would like to thank those who have contributed to the implementation of this research.

\section{REFERENCES}

Beyhaghi, M., \& Hawley, J. P. (2013). Modern portfolio theory and risk management: Assumptions and unintended consequences. Journal of Sustainable Finance \& Investment, 3(1), 17-37. https://doi.org/10.1080/20430795.2012.738600

Brimberg, J., Hansen, P., Laporte, G., Mladenović, N., \& Urošević, D. (2008). The maximum return-on-investment plant location problem with market share. Journal of the Operational Research Society, 59(3), 399-406. https://doi.org/10.1057/palgrave.jors.2602349

Duy Bui, L., Chi Le, T., Ngoc Quang, A. H., \& Wong, W.-K. (2021). Determinants of the possibilities by investors' risk-taking: Empirical evidence from Vietnam. Cogent $\begin{array}{llll}\text { Economics } \quad \text { F } & \text { Finance, } & 9(1), & \end{array}$ https://doi.org/10.1080/23322039.2021.1917106

Khalid, M., Abbas, Q., \& Nazir, M. S. (2020). Efficiently monitoring the ship of financially distressed companies sinking in Iron law of earnings management: Evidence from $\begin{array}{lllll}\text { Pakistan. Cogent Economics \& Finance, 8(1), } 1838685 . & \end{array}$ https://doi.org/10.1080/23322039.2020.1838685

Krylov, S. (2018). Target financial forecasting as an instrument to improve company financial health. Cogent Business \& Management, 5(1), 1540074. https://doi.org/10.1080/23311975.2018.1540074

Maidoki, P. B. (2013). Financial Reports and Managerial Effectiveness in Planning and Decision-making. Journal of Economics, 4(1), 69-73. https://doi.org/10.1080/09765239.2013.11884966 
Minh Trang, P. T., Tuan, N. M., \& Tho, N. H. (2017). The Role of Financial Ratios in the Variance of Stock Trading Volume in Emerging Stock Markets. Journal of AsiaPacific Business, 18(3), 180-191. https://doi.org/10.1080/10599231.2017.1346408

Mouna, A., \& Anis, J. (2016). Market, interest rate, and exchange rate risk effects on financial stock returns during the financial crisis: AGARCH-M approach. Cogent $\begin{array}{llll}\text { Economics } \quad \text { F } & 1125332 .\end{array}$ https://doi.org/10.1080/23322039.2015.1125332

Okunevičiūtė Neverauskienė, L., Tvaronavičienė, M., Rutkauskas, A. V., Danilevičienė, I., \& Stasytytè, V. (2021). The possibilities and consequences of investment decisions by stepwise optimization. Economic Research-Ekonomska Istraživanja, O(0), 1-27. https://doi.org/10.1080/1331677X.2021.1955222

Osiichuk, D., \& Mielcarz, P. (2021). The nonmonotonicity of cash-cash flow relationship: The role of uncertainty and financing constraints. Economic Research-Ekonomska Istraživanja, 34(1), 2263-2283. https://doi.org/10.1080/1331677X.2020.1862687

Ponikvar, N., Tajnikar, M., \& Pušnik, K. (2009). Performance Ratios for Managerial Decision-Making in a Growing Firm. Journal of Business Economics and Management, 10(2), 109-120. https://doi.org/10.3846/1611-1699.2009.10.109-120

Shakespeare, C. (2020). Reporting matters: The real effects of financial reporting on investing and financing decisions. Accounting and Business Research, 50(5), 425442. https://doi.org/10.1080/00014788.2020.1770928

Tran, K. H., \& Duong, N. H. (2020). Earnings management to avoid earnings decreases and losses: Evidence from Vietnamese listed companies. Cogent Economics \& Finance, 8(1), 1849980. https://doi.org/10.1080/23322039.2020.1849980

Xin, Q., Zhou, J., \& Hu, F. (2018). The economic consequences of financial fraud: Evidence from the product market in China. China Journal of Accounting Studies, 6(1), 1-23. https://doi.org/10.1080/21697213.2018.1480005 